Ann. Zootech., r973, $22(4), 463-484$.

\title{
INFLUENCES DES LIPIDES ALIMENTAIRES SUR LES MÉTABOLISMES CUPRIQUE ET MARTIAL CHEZ LE RAT DE LABORATOIRE
}

\author{
G. BOZZOLO, P. RAYNAUD et R. MONCOULON \\ Laboratoire des Productions animales, \\ École nationale supérieure agronomique \\ 145, avenue de Muret, \\ 31000 Toulouse
}

\section{RÉSUMÉ}

Des études expérimentales d'anémies provoquées chez le Rat ont été réalisées sur des lots de quinze animaux, à l'aide d'apports lipidiques alimentaires variés, en relation avec la régulation des métabolismes cuprique et martial. Les résultats montrent que l'intervention des lipides alimentaires, selon leur nature et leur composition en acides gras, s'exerce de façon différente sur l'hématopoièse. Cette intervention se situe notamment au niveau du stockage hépatique du fer et du cuivre dont dépendent les fluctuations de l'hématocrite et celles de l'excrétion fécale des oligo-éléments.

Le beurre laitier de vache favorise l'augmentation des réserves de fer dans le foie; le saindoux et l'huile de colza induisent une réduction du stock hépatique, l'huile de coprah, la lécithine du soja déterminent des valeurs intermédiaires. Le saindoux et l'huile de colza sont les seuls à provoquer l'apparition d'états anémiques.

\section{IN'TRODUCTION}

Il est classiquement admis que l'anémie physiologique du jeune, décrite par VAHLQUIST (I946) chez le nourrisson, résulte d'une carence martiale d'origine nutritionnelle due à la pauvreté des laits en fer. Les réserves foetales insuffisantes dans le foie, les apports alimentaires réduits, les effets de la dilution liée à la croissance intensive du jeune, constituent les causes majeures de l'apparition d'anémies normochromes microcytaires à évolution hypochrome.

Ces affections anémiques apparaissent spontanément dans l'élevage des jeunes animaux. Si elles sont recherchées systématiquement dans la production du veau de boucherie (ChARPENTIER, I966; MATRONE, et al., I957 ; EECKHOUT et al., I959 a 
et $b$ ), par contre elles sont néfastes chez le porcelet étant en relation avec la " crise des trois semaines ". La thérapeutique par injection de fer assimilable permet de rectifier ces anomalies.

Les théories de la carence primaire martiale ne sont pourtant pas suffisantes pour élucider le problème que posent de telles anémies. Les causes indirectes comme la carence secondaire par mauvaise absorption des oligoéléments indispensables à l'hématopoèìse ou même tertiaire par défaut d'utilisation métabolique sont plus probables (Bozzolo, I972). En effet, dans le contexte d'une alimentation située à la limite de la carence, les activateurs ou inhibiteurs de la dynamique martiale jouent un rôle majeur. Au contraire, leur action est amoindrie et ne se manifeste pas dans le cas d'une alimentation pléthorique. Parmi les nombreux facteurs susceptibles d'intervenir, les lipides alimentaires occupent, à notre avis, une place importante. Des travaux ont été réalisés pour déterminer leurs incidences sur l'absorption du fer. Hrrooka et al. (I968) ont montré, chez le Rat, que l'incorporation d'huile de soja dans la ration produit un effet bénéfique sur l'absorption du fer. Par contre, BRODAN et al. (I967), observent la diminution de l'assimilation intestinale du fer alimentaire en présence de lipides.

Par ailleurs, il est montré que l'apport alimentaire intervient indirectement sur la composition des globules rouges. La relation qui existe entre la fragilité du globule rouge et les lipides membranaires fait ressortir l'importance des apports lipidiques de la ration dans la diminution de la vie des érythrocytes (LoNGINI et Johnson, I943; Freeman et Johnson, I940 ; Aschkenasy et Blapin, I959; De Gier et Van Deenen, i96i ; Faust, igo8 ; Loewy, I943).

Ainsi les lipides alimentaires peuvent-ils déterminer l'apparition d'anémies hypochromes microcytaires (WATKIN, I957; LEVENSON et al., I957; MENG et FREEMAN, I948).

Pour ce qui nous concerne, nous nous posons la question de savoir si les lipides alimentaires n'interfèrent pas sur le rôle joué par les oligoéléments principaux, dans les processus hématopoiétiques.

\section{MATÉRIEL E'T MÉTHODES}

$$
\text { I. - Les animaux }
$$

Notre expérimentation est réalisée sur 8 lots de I 5 rats Wistar âgés de 40 jours au départ. Les animaux de ces lots sont nourris avec des régimes différents et sont sacrifiés $7^{\circ}$ jours après le début de l'expérimentation.

$$
\text { 2. - Les régimes }
$$

Nous utilisons comme régime témoin, sur le plan nutritionnel, un aliment commercial dont la composition est la suivante :

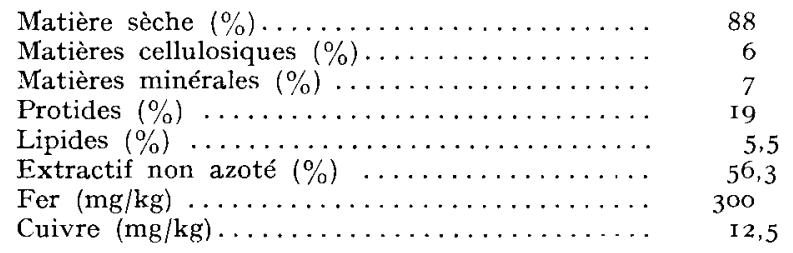




\section{Régimes expérimentaux.}

Tous ces régimes contiennent de la farine jaune de maïs dégermé, retenue pour sa pauvreté en matières grasses et sa richesse en caroténoïdes. Ce composant nous permet en outre de confectionner les aliments à $43 \mathrm{p}$. roo de MS sous forme de galettes. Les différents régimes sont établis à partir de la ration de base dont la formule est donnée au tableau $I$.

\section{TABI,EAU I}

Composition en matières organiques du régime de base

\begin{tabular}{c|c|c|c}
\hline & \multicolumn{3}{|c}{ Apports nutritifs } \\
\cline { 2 - 4 } Composants & $\begin{array}{c}\text { Matières } \\
\text { grasses } \\
(\mathrm{g})\end{array}$ & $\begin{array}{c}\text { Matières } \\
\text { protéiques } \\
(\mathrm{g})\end{array}$ & $\begin{array}{c}\text { Matières } \\
\text { non azotées } \\
(\mathrm{g})\end{array}$ \\
\hline $\begin{array}{c}\text { Lait écrémé en poudre } 200 \mathrm{~g} \\
\text { I'arine de maïs dégermée } \\
600 \mathrm{~g}\end{array}$ & 2 & 68 & 98 \\
Caséine lactique $80 \mathrm{~g}$ & 6 & 52 & 100 \\
Saccharose $100 \mathrm{~g}$ & & 80 & 450 \\
Solution minérale $20 \mathrm{~cm}^{3}$ & & & \\
\hline \hline
\end{tabular}

Il est ajouté aux composants organiques $20 \mathrm{ml}$ de la solution minérale suivante :

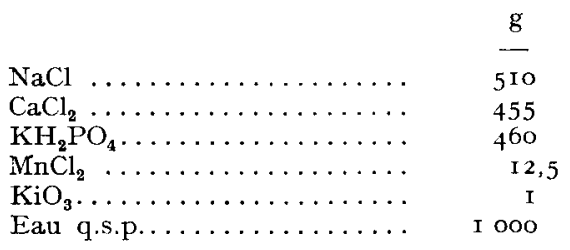

TABLEAU 2

Composition minérale de base après adjonction de la solution minérale et compte tenu de la teneur minérale des différents composants exprimée en p.p.m.

\begin{tabular}{|c|c|c|c|c|}
\hline $\begin{array}{c}\text { Nature } \\
\text { des éléments }\end{array}$ & $\begin{array}{c}\text { Apport } \\
\text { par le maïs }\end{array}$ & $\begin{array}{c}\text { Apport } \\
\text { par le lait }\end{array}$ & $\begin{array}{c}\text { Apport } \\
\text { par l'additif }\end{array}$ & $\begin{array}{l}\text { Composition } \\
\text { finale }\end{array}$ \\
\hline Sodium & 60 & 700 & 4000 & 5000 \\
\hline Iode $\ldots \ldots \ldots$ & & 0,03 & 0,70 & 0,73 \\
\hline Manganèse & 3 & 0,4 & 70 & 75 \\
\hline Calcium....... & 100 & 1600 & 3300 & 5000 \\
\hline Phosphore ... & 1500 & 1400 & 2100 & 5000 \\
\hline Potassium.... & 1600 & 2100 & 3000 & 6700 \\
\hline Magnésium ... & 600 & 140 & & $7: 0$ \\
\hline
\end{tabular}


Pour ce qui concerne les oligoéléments, nous avons vérifié la teneur en fer et en cuivre de la ration de base utilisée. L'analyse fait ressortir une teneur de 53, Ip.p.m. en fer et de 2,I p.p.m. en cuivre. La prise alimentaire, étant supérieure à I $2 \mathrm{~g}$ de matière sèche, correspond donc à une ingestion de $0,700 \mathrm{mg}$ de fer et de $0,03 \mathrm{mg}$ de cuivre /jour/rat.

L'eau de boisson fournit également un apport supplémentaire que nous avons évalué à $0,008 \mathrm{mg}$ de fer et $0,005 \mathrm{mg}$ de cuivre pour les mêmes conditions.

On peut de ce fait estimer que les besoins physiologiques en oligo-éléments sont couverts puisque les apports dépassent pour un rat de $70 \mathrm{~g}$ par jour, les valeurs de o,5 $\mathrm{mg}$ de fer et de $0,02 \mathrm{mg}$ de cuivre (Muntwyller et Hanzel, I933 ; BonN, I966; Matrone et al., I957; McCall et al., I962; UNDERWOOD, I962; UNDRITZ, 1964).

\section{Régimes constitués à partir de la ration de base.}

Les régimes ne diffèrent que par la nature des sources lipidiques. Dans tous les cas celles-ci sont ajoutées à raison de $\mathrm{I}$ oo $\mathrm{g} / \mathrm{kg}$ de ration de base. La numérotation des différents lots d'animaux, qui est faite en fonction de l'attribution des régimes, est la suivante :

- lot I : régime à la lécithine du soja, retenu pour sa richesse en acide linoléique ;

- lot 2 : régime à l'huile de coprah, riche en acide laurique ;

- lot 3 : régime au saindoux, source appréciable en acides gras saturés à longue chaîne comme les acides palmitique et stéarique;

- lot 4 : régime à l'huile de colza contenant de l'acide érucique en grande quantité ;

- lot 5 : régime au beurre laitier d'origine bovine présentant l'avantage d'être une source de matières grasses riches en acides gras à courtes chaînes ;

- lot 6 : régime lipidoprive ;

- lot T: Alimentation du commerce pour rats d'élevage.

$$
\text { 3. - Méthodes }
$$

\section{a) Les prélèvements sanguins.}

Les rats sont anesthésiés à l'éther pour éviter certaines variations des constituantes sanguines dues à des mécanismes de stress capables de provoquer les décharges spléniques (KAYSER, I963).

Les prélèvements sont effectués au niveau d'une veine caudale.

b) Hématocrite. 1968).

L'hématocrite est évalué par la méthode du " micro-hématocrite " (RoDriguez, BrogLIo,

c) Numération globulaive.

La méthode de numération adoptée est celle préconisée par Pinto et BaLdr (I968) qui utilisent le principe des lois colorimétriques de BEER-LAMBERT avec des dilutions adéquates dans la solution physiologique de Burlina. La longueur d'onde choisie au spectrophotomètre Coleman Junior est de $525 \mathrm{~m} \mu$.

d) Les fèces.

Les fèces sont recueillies tous les jours pendant une semaine pour chaque lot d'animaux. L'analyse est faite à partir d'un échantillon représentatif des fèces " hebdomadaires " préalablement desséchées à l'étuve à ro $5^{\circ} \mathrm{C}$.

e) Les prélèvements d'organes.

Les animaux sont saignés à blanc par décapitation afin de favoriser la vidange des réserves sanguines organiques (KENT et al., I963). Le foie, la rate, les reins sont prélevés. Le foie est ensuite perfusé à l'aide de liquide physiologique dans le but d'évacuer un reliquat éventuel d'hématies.

\section{f) Méthodes chimiques.}

Les minéralisations employées sont de deux types :

- minéralisation par voie sèche au four électrique à $55^{\circ} \mathrm{C}$ pendant $\mathrm{i} 2 \mathrm{~h}$ pour les fèces,

- minéralisation par voie humide pour les organes avec le mélange sulfo-nitrique (LoISELEUR, I963).

Les dosages du fer et du cuivre sont effectués par absorption atomique sur appareil PerkinElmer P 290 avec des cathodes spécifiques pour chacun des éléments à la longueur d'onde $\lambda$ : $364 \mathrm{~m} \mu$ pour le fer et à $\lambda$ : $290 \mathrm{~m} \mu$ pour le cuivre. 
g) Méthodes d'analyse mathématique.

L'analyse statistique des résultats est conduite d'après la comparaison des variances selon des plans expérimentaux à plusieurs facteurs. Elle fait aussi appel aux méthodes de la covariance. Les résultats sont accompagnés des tableaux d'analyse de la variance.

\section{RÉSULTATS}

\section{1. - Croissance pondérale des animaux}

Les animaux d'un même lot sont pesés au même moment. Ainsi cinq pesées ont été faites sur l'animal pendant la durée de l'expérience. Les valeurs pondérales relevées ont permis d'établir les courbes de croissance représentées dans la figure $I$.

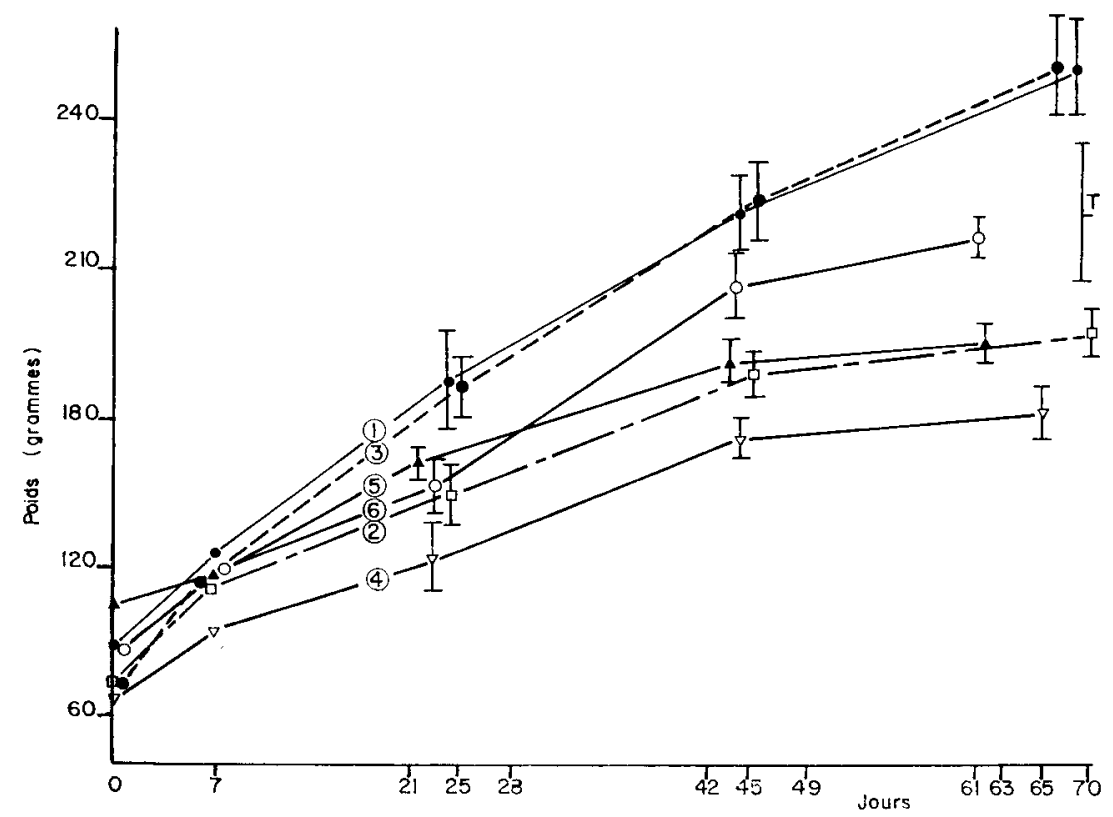

FIG. I. - Évolution du poids en fonction du temps selon les régimes alimentaires

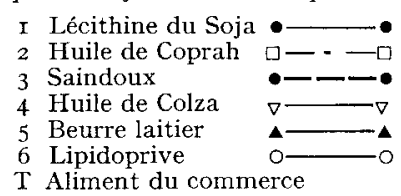

De l'examen de cette figure, il ressort les points suivants :

- le lot 6 (régime lipidoprive) présente des gains pondéraux normanx en référence du lot $\mathrm{T}$ (aliment du commerce), les différences de poids entre les animaux de ces deux lots ne sont pas significatives (à $p .0,05$ ),

- les lots 5 (supplémenté avec du beurre), 2 (supplémenté avec de 1'huile 
de coprah), présentent une prise de poids plus modérée. Cela se manifeste de façon significative I mois après le début de l'expérience. Cet effet est d'ailleurs plus accentué et plus précoce pour le lot 4 (supplémenté avec de l'huile de colza),

- le lot 3 (régime au saindoux) et le lot I (régime à la lécithine du soja) font apparaître, à l'inverse, un accroissement pondéral significativement supérieur, dès le Io $^{\mathrm{e}}$ jour après le début de l'expérimentation.

\section{2. - Évolution de l'hématocrite}

L'évolution de l'hématocrite a été établie à partir de quatre séries de mesures réalisées à différents temps de l'expérimentation. Les résultats sont interprétés au moyen d'un plan statistique d'ordre 2 à 55 répétitions. Le tableau 3 rend compte des différentes données acquises.

TABLEAU 3

Résumé de l'analyse de la variance velative aux fluctuations de l'hématocrite

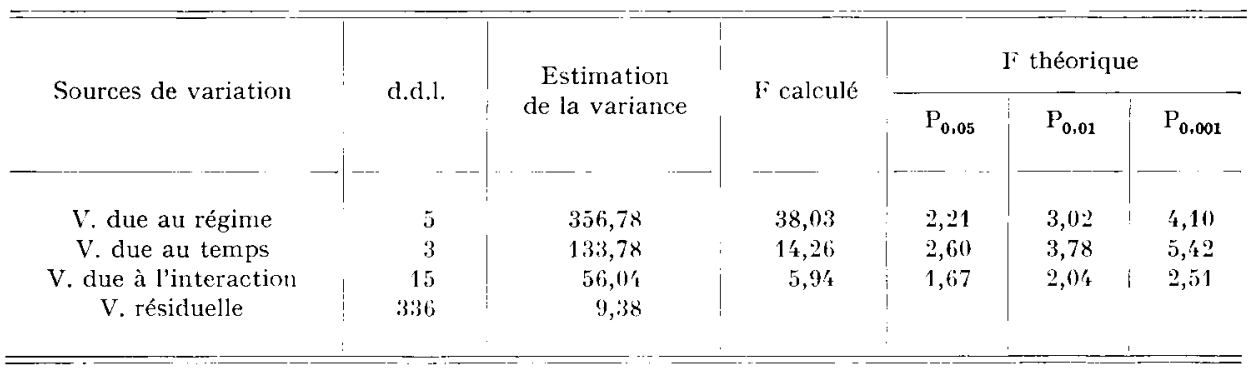

Des résultats obtenus il ressort que les effets des facteurs : "temps ", "régime" et " interaction" montrent une influence hautement significative sur l'évolution de l'hématocrite, même à la probabilité d'erreur : I p. I 000.

\section{a) Effet des facteurs temps et interaction.}

L'analyse de la variance met en évidence une intervention de ces paramètres sur l'expression de l'hématocrite. Par comparaison avec la p.p.d.s. $\left(\Delta \mathrm{P}_{0,05}=0,87\right)$, la période des 20 premiers jours se caractérise par une diminution de l'hématocrite, alors que la phase terminale est marquée par un relèvement de sa valeur. L'incidence du facteur temps ressort d'ailleurs de l'examen de la figure 2 : elle intéresse l'ensemble des données. Il n'est pas évident que le choix de la nature des régimes n'intervienne pas dans cet effet. C'est ce qu'exprime l'influence de l'interaction, estimée significative par le test F. La variation de l'hématocrite est assujettie au cours du temps à la nature du régime alimentaire.

b) Effet du facteur régime.

La comparaison des moyennes deux à deux avec l'expression de la plus petite différence significative calculée (p.p.d.s.) à $\mathrm{P}_{0,05}(\Delta=I, 09)$ montre que les lots diffèrent significativement de la façon suivante : 


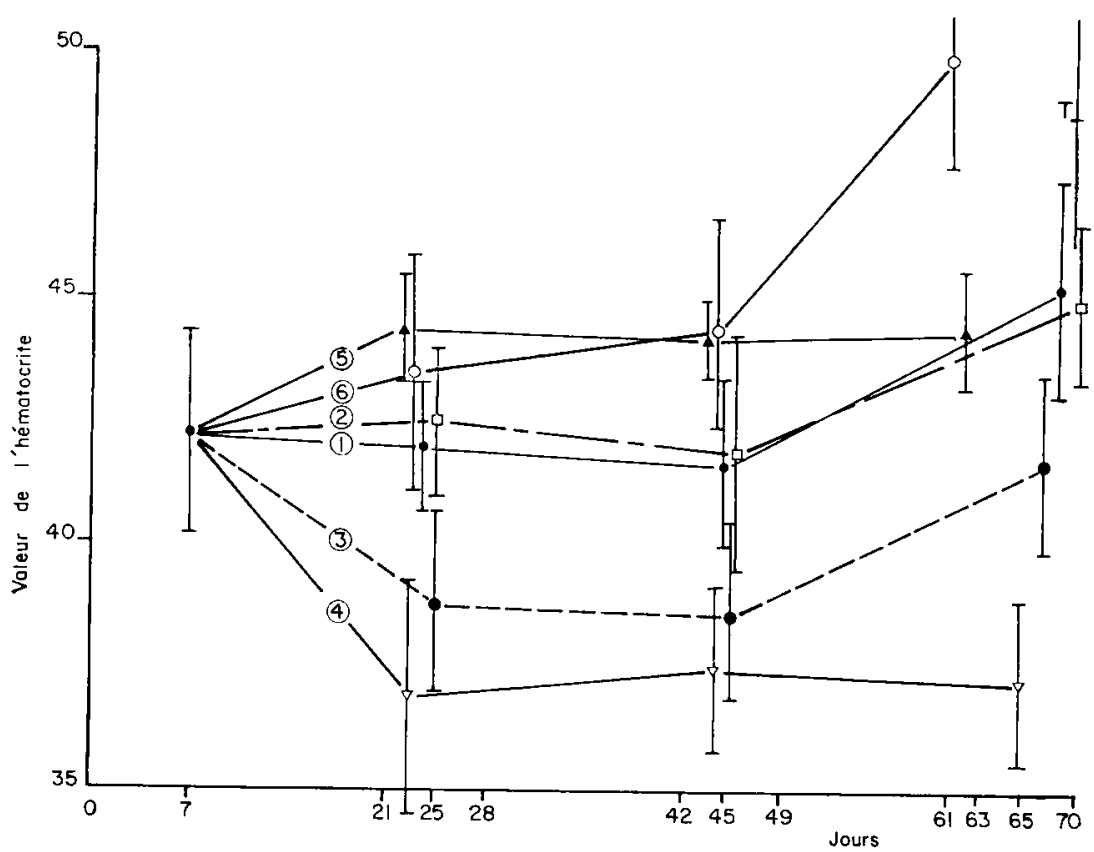

Frg. 2. - Évolution de la valeur de l'hématocrite en fonction du temps selon les régimes alimentaires

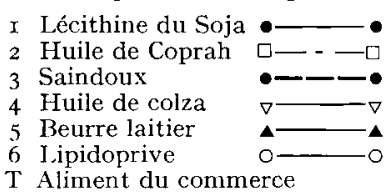

Les lots I (régime à 1a lécithine du soja), 2 (régime à 1'huile de coprah) et 5 (régime au beurre laitier) évoluent de façon semblable pendant toute la période expérimentale. Il en va différemment pour les lots 3, 4 et 6 qui se caractérisent par une évolution bien individualisée. Alors que les matières grasses du beurre (lot 5), de l'huile de coprah (lot 2) et de la lécithine du soja (lot I) induisent une certaine constance dans la valeur de l'hématocrite, le régime lipidoprive (lot 6) s'accompagne d'une augmentation de cette valeur. Pour ce dernier régime la valeur terminale de l'hématocrite est la même que celle obtenue pour les animaux nourris avec l'aliment du commerce (lot $\mathrm{T}$ ).

L'alimentation complémentée au saindoux (lot 3) entraîne une diminution précoce des valeurs de l'hématocrite. Cet effet n'est pas définitif car une remontée discrète de ce dernier se dessine à la fin de l'expérience. La diminution précoce est accentuée avec le régime à l'huile de colza (lot 4) mais dans ce cas on n'observe pas. de remontée.

\section{3. - Évolution de la numération globulaire}

Trois numérations globulaires ont été réalisées aux temps suivants : début d'expérimentation, 25 jours et 70 jours après. 


\section{TABLEAU 4}

Résumé de l'analyse de la variance relative aux fluctuations de la numération globulaire

\begin{tabular}{|c|c|c|c|c|c|c|}
\hline \multirow{2}{*}{ Sources de variation } & \multirow{2}{*}{ d.d.l. } & \multirow{2}{*}{$\begin{array}{c}\text { Estimation } \\
\text { de la variance }\end{array}$} & \multirow{2}{*}{ F calculé } & \multicolumn{3}{|c|}{ F théorique } \\
\hline & & & & $P_{0,05}$ & $\mathrm{P}_{0,01}$ & $P_{0,001}$ \\
\hline $\begin{array}{c}\text { V. due au temps } \\
\text { V. due aux régimes } \\
\text { V. due à l'interaction } \\
\text { V. résiduelle }\end{array}$ & $\begin{array}{r}2 \\
5 \\
10 \\
252\end{array}$ & $\begin{array}{r}495,292670 \\
17,681446 \\
12,044249 \\
7,696480\end{array}$ & $\begin{array}{r}64,30 \\
2,21 \\
1,56\end{array}$ & $\begin{array}{l}3,00 \\
2,21 \\
1,83\end{array}$ & 3,78 & 6,91 \\
\hline
\end{tabular}

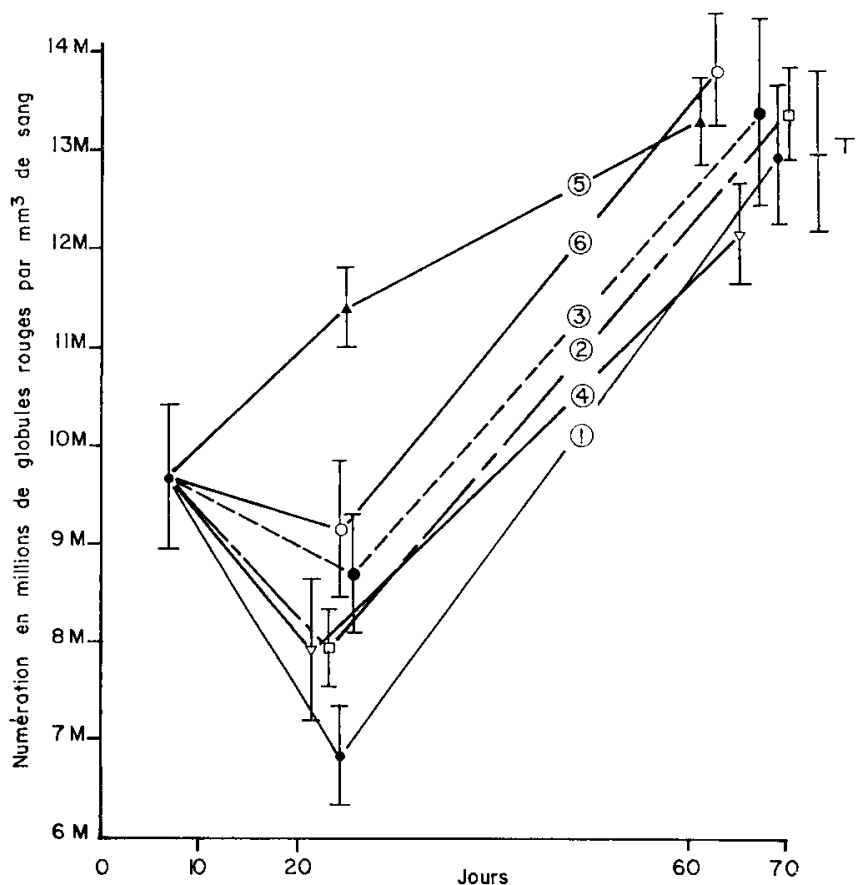

FIG. 3. - Évolution de la mumération globulaive avec le temps selon la nature des régimes

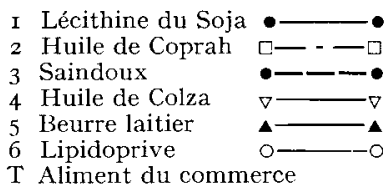

Le tableau concernant l'analyse de la variance et le graphique de la figure 3 permettent de faire ressortir les points suivants :

On assiste à une évolution significative à $\mathrm{P}_{0,001}$ de la numération globulaire pendant la durée de l'expérimentation et ceci indépendamment de la nature de 
régimes. Cette évolution est caractérisée aux environs du $25^{\mathrm{e}}$ jour par une tendance à la diminution du nombre de globules rouges, suivie d'une remontée qui se poursuit jusqu'à la fin de notre expérimentation. A la probabilité d'erreur de $5 \mathrm{p}$. roo, la diminution de la valeur globulaire au $25^{\mathrm{e}}$ jour n'est pas la même en fonction du régime : inexistante avec le régime au beurre laitier, elle est accentuée avec le régime à la lécithine du soja (lot $\mathrm{I}$ ). L'augmentation qui s'opère pour tous les régimes à partir de cette période aboutit à des valeurs qui ne sont plus significativement distinctes. Ceci est confirmé par l'analyse de la variance qui se situe à la limite de la signification. La comparaison avec la p.p.d.s. ne laisse pas apparaître, à ce stade, de différences significatives entre régimes. On ne décèle pas d'incidence imputable à 1" "interaction ".

\section{4. - Analyse de l'infuence des lipides alimentaires \\ sur les réserves martiale et cuprique des organes du rat}

a) Réserves martiales.

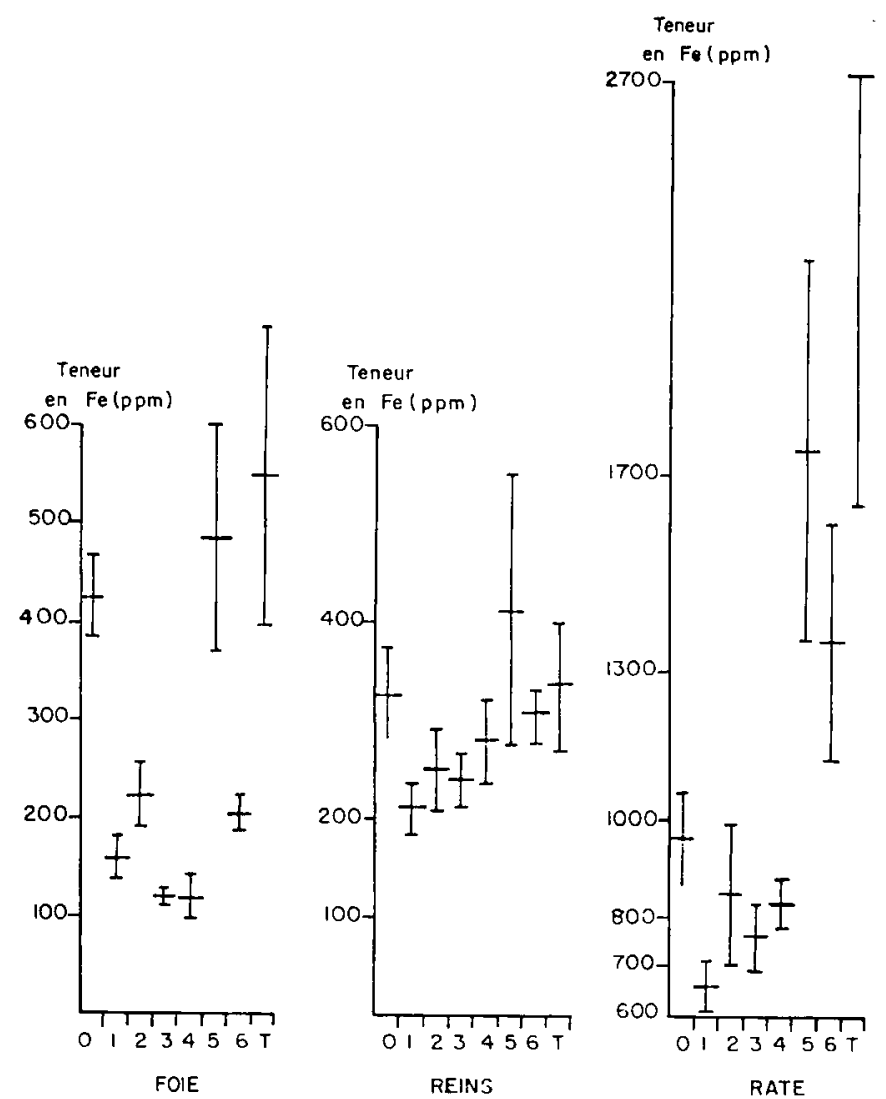

Frg. 4. - Teneurs en Fer des organes du Rat selon le régime alimentaire

- Début d'expérience

I Lécithine du Soja

2 Huile de Coprah

3 Saindoux

4 Huile de Colza

5 Betrre laitier

6 Lipidoprive

$\mathrm{T}$ Aliment du commerce 
TABLEAU 5

Résumé de l'analyse de la variance velative aux fuctuations des réserves martiales dans les organes des rats

\begin{tabular}{|c|c|c|c|c|c|c|c|}
\hline \multirow{2}{*}{\multicolumn{2}{|c|}{ Sources de variation }} & \multirow{3}{*}{$\begin{array}{c}\text { d.d.1. } \\
-- \\
5 \\
84\end{array}$} & \multirow{3}{*}{$\begin{array}{l}\text { Estimation } \\
\text { de la variance } \\
\begin{array}{r}280662,26 \\
7467,22\end{array}\end{array}$} & \multirow{3}{*}{$\begin{array}{c}\text { F calculé } \\
37,58\end{array}$} & \multicolumn{3}{|c|}{ F théorique } \\
\hline & & & & & & & \\
\hline Foie & $\begin{array}{l}V \text {. due aux régimes } \ldots \\
V \text {. aléatoire } \ldots \ldots\end{array}$ & & & & 2,33 & 3,26 & 4,27 \\
\hline Rate & $\begin{array}{l}V \text {. due aux régimes } \ldots \\
V \text {. aléa toire } \ldots \ldots \ldots\end{array}$ & $\begin{array}{r}5 \\
8 ! \\
8\end{array}$ & $\begin{array}{r}2717042,05 \\
107677,23\end{array}$ & 25,2 & 2,33 & $3, \geq 6$ & 4,27 \\
\hline Reins & $\begin{array}{l}\text { V. due aux régimes } \ldots \\
V . \text { aléa toire .......... }\end{array}$ & $\begin{array}{r}5 \\
81\end{array}$ & $\begin{array}{l}76271,86 \\
32417,34\end{array}$ & 2,35 & 2,33 & 3,26 & 4,27 \\
\hline
\end{tabular}

\section{a. Foie.}

Dans le foie, le test du $\mathrm{F}$ est hautement significatif en ce qui concerne l'influence des régimes alimentaires sur la concentration martiale. L'estimation de la p.p.d.s. à la probabilité d'erreur de I p. I ooo $(\Delta=$ I04,0 p.p.m.), montre par comparaison de 1 ' " effet régime " que le régime riche en beurre (lot 5) se comporte de façon totalement indépendante des autres groupes; il détermine la plus forte teneur martiale (deux à trois fois plus élevée). Par contre, il n'existe pas de différence significative entre les effets des régimes à l'huile de colza (lot 4) et au saindoux (lot 3), attendu que dans les deux cas on enregistre les valeurs les plus faibles. Le groupe constitué par les lots 2 (régime à l'huile de coprah), I (régime à la lécithine du soja) et 6 (régime lipidoprive) occupent une position intermédiaire, significativement distincte.

\section{B. Rate.}

L'analyse de la variance par le test $\mathrm{F}$ laisse supposer que les lipides alimentaires ont une incidence significative sur les concentrations en fer.

La comparaison des différences à la p.p.d.s. $\left(\Delta \mathrm{P}_{\mathbf{0}, 05}=234,6\right.$ p.p.m. $)$ indique trois positions significativement distinctes. La plus basse est occupée par les lots : I (lécithine du soja), 2 (huile de coprah), 3 (saindoux) et 4 (huile de colza) (environ 800 p.p.m.). Le lot 6 (lipidoprive) arrive en seconde position (environ I 300 p.p.m.). Les régimes au beurre laitier (lot 5) (I 700 p.p.m.) et à l'aliment du commerce (lot T) se situent aux plus hauts niveaux.

\section{$\gamma$. Reins.}

La comparaison deux à deux avec la p.p.d.s. $\left(\Delta \mathrm{P}_{\mathbf{0}, 05}=\mathrm{I} 29\right.$ p.p.m.) fait ressortir que seul le régime au beurre laitier (40o p.p.m.) se comporte de façon significativement différente de tous les autres. L'ensemble des lots I, 2, 3 et 4 est fortement regroupé autour de la côte $25^{\circ}$ p.p.m. Le lot 6 occupe une position intermédiaire. La concentration du fer dans le tissu rénal, au début et à la fin de l'expérience, exprimée pour les animaux des lots o et $T$ ne varie pas et demeure voisine de celle du régime au beurre laitier (lot 5 ). 
b) Réserves cupriques.

๙. Foie.

Au niveau hépatique l'analyse de la varrance traduit une influence hautement significative de la nature des régimes sur la mise en réserve du cuivre, le calcul de la p.p.d.s. permet par comparaison $\left(\Delta \mathrm{P}_{0,05}=\mathrm{I}, 3\right.$ p.p.m. et $\Delta \mathrm{P}_{0,001}=2,2$ p.p.m. $)$, d'affirmer que le régime à l'huile de coprah (lot 2) se comporte de façon différente de tous les autres régimes au risque d'erreur de I p. I ooo. Les animaux de ce lot
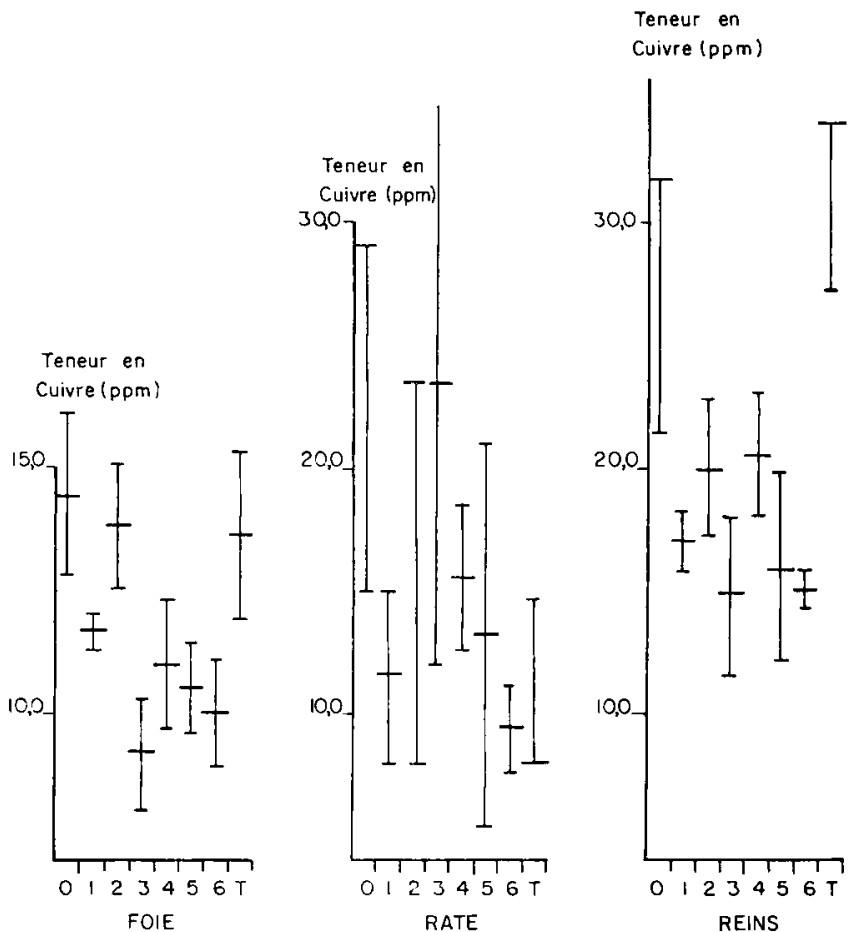

FIG. 5. - Teneur moyenne en Cuivre dans les organes du rat selon le régime alimentaire
- Début d'expérience
I Lécithine du Soja
2 Huile de Coprah
3 Saindoux
4 Huile de Colza
5 Beurre laitier
6 Lipidoprive
T Aliment du commerce

détiennent la valeur cuprique hépatique la plus élevée avec ceux des lots $T$ et o (environ I4 p.p.m.). Parmi les autres régimes, seuls les lots complémentés à la lécithine du soja (I) et au saindoux (3) présentent entre eux une différence significative.

A la probabilité d'erreur de 5 p. Ioo on fait apparaître des différences significatives supplémentaires. Le lot 3 se distingue du groupe 5 et 4 , de même que le lot 6 régime lipidoprive) du lot I (régime à la lécithine du soja). 


\section{TABLEAU 6}

Résumé de l'analyse de la variance relative aux fluctuations des réserves cupriques dans les organes du rat

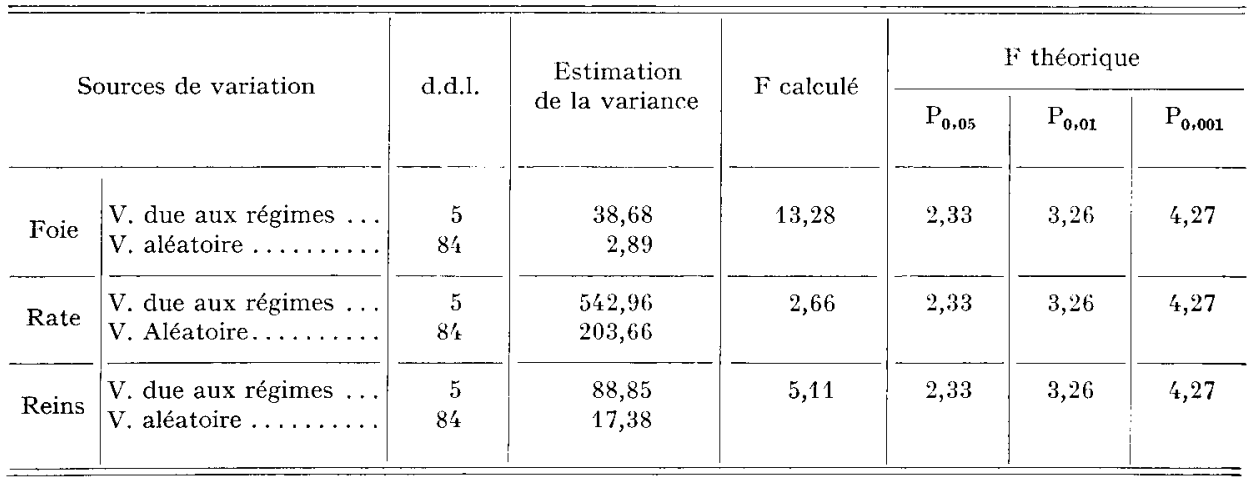

\section{ß. Rate.}

L'étude des réserves spléniques ne révèle qu'une influence restreinte du facteur alimentaire, à la limite de la signification pour une probabilité d'erreur de $5 \mathrm{p}$. Ioo.

Dans ces conditions la p.p.d.s. ( $\Delta=$ I0,2 p.p.m.) fait ressortir que l'influence des régimes au saindoux (lot 3 ) et à l'huile de coprah (lot 2 ) (teneur hépatique = 23 p.p.m.) est différente de celle exercée par les régimes lipidoprive (6) et à la lécithine du soja (lot $\mathrm{I}$ ) (th = Io p.p.m.). Nous pouvons également faire remarquer qu'en début d'expérience et avant la distribution des différents régimes, la concentration hépatique cuprique est élevée (29 p.p.m.). C'est avec le régime du commerce (lot T) que l'effondrement est le plus marqué au terme de l'expérience (th $=8$ p.p.m.).

\section{Ү. Reins.}

On retrouve pour les reins comme pour le foie une influence du régime sur l'importance des réserves.

A la marge d'erreur de $5 \mathrm{p}$. Ioo la distribution des concentrations se fait de la façon suivante : les lots o et $T$ possèdent les valeurs les plus élevées avec 32 et 34 p.p.m., le groupe des lots 2 et 4 vient ensuite et de façon significative avec environ une teneur rénale de 20 p.p.m., enfin l'ensemble des lots 6,5 et 3 présente la plus faible concentration en cuivre de 15 p.p.m.

\section{5. - Infuence des régimes alimentaires sur l'excrétion fécale du fer et du cuivre.}

L'analyse statistique pour les deux éléments est conduite selon un plan factoriel d'ordre 2 à une répétition.

a) Évolution de l'excrétion martiale dans les fèces.

D'après ce tableau, le facteur " temps " et le facteur " nature des régimes " possèdent tous deux une influence significative sur l'évolution de l'excrétion fécale. 


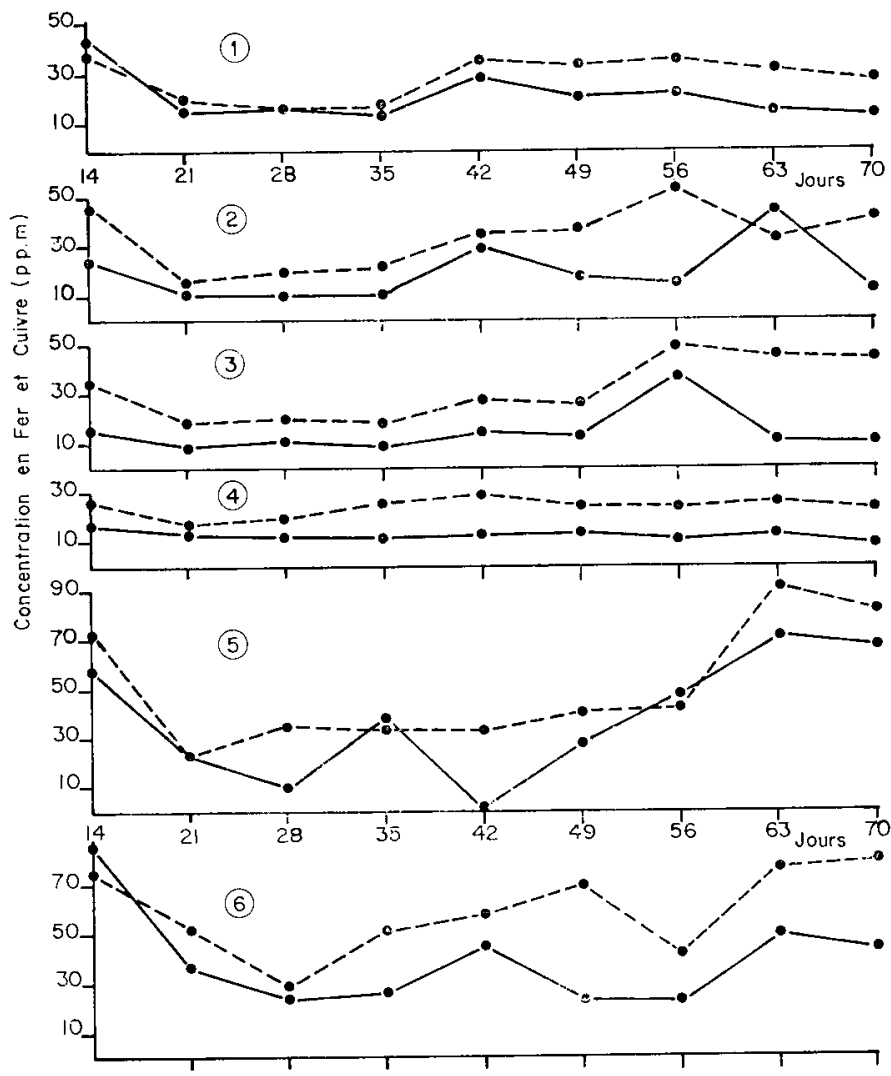

FIG. 6. - Evolution de la teneur en fer et en cuivre dans les fèces en fonction du temps et des régimes

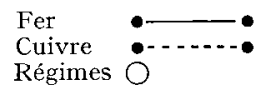

\section{TABLEAU 7}

Résumé de l'analyse de la variance relative aux fuctuations de l'excrétion martiale dans les fèces

\begin{tabular}{|c|c|c|c|c|c|c|}
\hline \multirow{2}{*}{ Sources de variation } & \multirow{2}{*}{ d.d.l. } & \multirow{2}{*}{$\begin{array}{c}\text { Estimation } \\
\text { de la variance }\end{array}$} & \multirow{2}{*}{ F calculé } & \multicolumn{3}{|c|}{$\mathrm{F}$ théorique } \\
\hline & & & & $P_{0,01}$ & $P_{0,05}$ & $P_{0,001}$ \\
\hline $\begin{array}{l}\text { V. due au temps } \\
\text { V. due aux régimes } \\
\text { V. résiduelle }\end{array}$ & $\begin{array}{r}5 \\
8 \\
40\end{array}$ & $\begin{array}{r}2647,65 \\
45,84 \\
89,96\end{array}$ & $\begin{array}{r}29,43 \\
5,09\end{array}$ & $\begin{array}{l}2,45 \\
2,18\end{array}$ & $\begin{array}{l}3,51 \\
2,99\end{array}$ & $\begin{array}{l}5,15 \\
4,21\end{array}$ \\
\hline
\end{tabular}




\section{$\alpha$. Influence des régimes.}

Le calcul de la p.p.d.s. $\left(\Delta \mathrm{P}_{0,05}=8,6\right.$ p.p.m. $)$ fait ressortir que c'est le régime au beurre laitier qui détermine la plus forte excrétion martiale, suivi dans cet effet par le régime lipidoprive. Les autres régimes favorisent une excrétion plus faible et on ne peut faire de distinction entre eux.

\section{Influence du temps.}

La mesure du fer fécal au cours du temps fait apparaître par comparaison avec la p.p.d.s. $\left(\Delta \mathrm{P}_{0,05}=\mathrm{I} 0,6\right.$ p.p.m. $)$, une évolution de l'excrétion irrégulière qui se traduit de la façon suivante :

- d'après les dosages effectués au $20^{\mathrm{e}}$ jour il apparaît une diminution significative de l'excrétion martiale,

- après 1 le $20^{\mathrm{e}}$ jour et jusqu'au $57^{\mathrm{e}}$ jour les valeurs relevées ne sont pas significativement différentes,

- aux mesures effectuées au $63^{\mathrm{e}}$ jour il apparaît une augmentation significative de l'excrétion martiale qui conduit à des valeurs voisines de celle du départ expérimental,

- aux environs du $7^{\mathrm{e}}$ jour l'excrétion du fer a diminué.

b) Évolution de l'excrétion cuprique dans les fèces.

\section{$\alpha$. Facteur régime.}

De l'analyse de la variance à $\mathrm{P}_{0,001}$ il ressort une incidence de l'alimentation lipidique sur l'excrétion fécale du cuivre.

Aux risques $P_{0,05}$ et $P_{0,001}\left(\Delta P_{0,05}=9,3\right.$ p.p.m. et $\Delta P_{0,001}=15,6$ p.p.m. $)$, on peut distinguer deux sortes de régimes:

- un premier groupe constitué par les régimes au beurre laitier (lot 5) et lipidoprive (lot 6), favorise une excrétion cuprique élevée,

- un detuxième groupe qui comprend l'ensemble des lots I, 2, 3, 4, détermine une excrétion cuprique inférieure.

\section{TABLEAU 8}

\section{Résumé de l'analyse de la variance relative aux fluctuations} de l'excrétion cuprique dans les fèces

\begin{tabular}{|c|c|c|c|c|c|c|}
\hline \multirow{2}{*}{ Sources de variation } & \multirow{2}{*}{ d.d.l. } & \multirow{2}{*}{$\begin{array}{c}\text { Estimation } \\
\text { de la variance }\end{array}$} & \multirow{2}{*}{ IF calculé } & \multicolumn{3}{|c|}{ F théorique } \\
\hline & & & & $P_{0,05}$ & $P_{0,01}$ & $P_{0,001}$ \\
\hline V. due au temps & 5 & 2327,67 & 18,29 & 2,45 & 3,51 & 5,15 \\
\hline$V$. due aux régimes & 8 & 567,52 & 4,46 & 2,18 & 2,99 & 4,21 \\
\hline V. aléatoire & 40 & 127,26 & & & & \\
\hline
\end{tabular}

\section{B. Facteur "temps».}

Avec le risque d'erreur de I p. I ooo on constate que l'excrétion fécale du cuivre est la même avant le $14^{\mathrm{e}}$ jour et après le $64^{\mathrm{e}}$ jour. Entre ces deux dates on observe 
une diminution qui ne devient significative qu'entre $2 \mathrm{I}$ et 35 jours, après le début de l'expérience.

Avec le risque d'erreur de $5 \mathrm{p}$. Ioo, on retrouve une évolution de l'excrétion similaire mais on peut fractionner la période comprise entre le $\mathrm{I} 4^{\mathrm{e}}$ et le $5^{6^{\mathrm{e}}}$ jour. C'est ainsi que l'on peut distinguer un stade situé entre le $2 \mathrm{I}^{\mathrm{e}}$ et le $28^{\mathrm{e}}$ jour, pendant lequel le niveau est le plus bas et un stade compris entre le $42^{\mathrm{e}}$ et le $49^{\mathrm{e}}$ jour caractérisé par le début de la remontée de l'excrétion.

\section{DISCUSSION}

L'examen des résultats que nous avons obtenus nous amène à émettre un certain nombre d'hypothèses concernant l'influence des lipides de la ration sur les anémies provoquées, en relation avec les métabolismes du fer et du cuivre.

\section{L'incidence sur l'anémie}

La comparaison des effets respectifs d'un régime lipidoprive et des régimes contenant des lipides fait ressortir que les graisses utilisées, quelle que soit leur nature, déterminent un hématocrite toujours inférieur à celui qu'induit un régime sans graisse. En ce qui concerne la comparaison avec l'effet de l'alimentation commerciale dont nous considérons l'influence comme normale, puisque l'hématocrite des animaux de ce lot est voisin de 46 , norme reconnue chez cette espèce animale, l'influence des sources lipidiques est variable. Nous rappelons que seuls les hématocrites significativement les plus bas, sont obtenus avec les rations contenant de l'huile de colza et du saindoux. Ces deux régimes possèdent donc un pouvoir anémiant. Dans les deux cas les valeurs de la numération globulaire sont, pour la durée de notre expérimentation, semblables à celle des autres régimes y compris le régime lipidoprive. Il est donc naturel de penser que les effets du saindoux et de l'huile de colza tendent à installer une anémie du type normochrome microcytaire.

Pourtant la qualification de ce type d'anémie ne rend pas compte des comportements différents des animaux nourris avec ces deux composants lipidiques. Les rats alimentés avec la ration au saindoux ont eu une croissance plus importante et ont présenté un état d'engraissement plus marqué. De plus, nous avons pu déceler chez ces animaux, une tendance à l'asthénie. On note par contre, que les animaux nourris avec la ration à l'huile de colza ont présenté, outre une croissance plus lente, un comportement anormal assez voisin d'un état pathologique.

\section{L'excrétion fécale}

Nous rappelons que tous nos régimes apportent une quantité suffisante d'oligoéléments : notre expérimentation ne se trouve donc nullement dans des conditions de carence.

- En premier lieu, nous constatons que les excrétions martiale et cuprique évoluent dans le même sens en fonction de la nature des régimes. Le coefficient de corrélation calculé $r=0,83$ au risque $\alpha=0$,or montre que la liaison entre fer et cuivre reste très étroite ( $r$ théorique $=0,267)$. 
Le rapport fer/cuivre demeure d'ailleurs le même pour les différents lots (o,64 $\pm 0,18)$, l'analyse de la variance résumée au tableau 9 n'étant pas significative. Nous pouvons donc considérer, qu'au cours de l'expérience, l'absorption du fer et du cuivre varient dans le même sens.

\section{TABLEAU 9}

Résumé de l'analyse de la variance du rapport fer/cuivre dans les fèces en fonction des différents régimes

\begin{tabular}{c|c|c|c|c}
\hline $\begin{array}{c}\text { Origine } \\
\text { de la variation }\end{array}$ & d.d.l. & Variance & F calculé & $\begin{array}{c}\text { F théorique } \\
\text { à P } 0,05\end{array}$ \\
\hline $\begin{array}{c}\text { V. due au régime } \\
\text { V. aléatoire }\end{array}$ & 5 & 0,794 & 1,27 & 2,45 \\
\hline
\end{tabular}

- En second lieu, il nous a paru intéressant de rapprocher les valeurs de l'excrétion des oligo-éléments étudiés des valeurs de l'hématocrite pour les différents lots.

Ce rapprochement fait ressortir que les variations se font de façon similaire : à l'hématocrite élevé correspond une excrétion de fer également élevée comme l'indique la figure 7. A l'opposé, les valeurs les plus faibles de l'excrétion fécale se rencontrent pour les hématocrites bas.
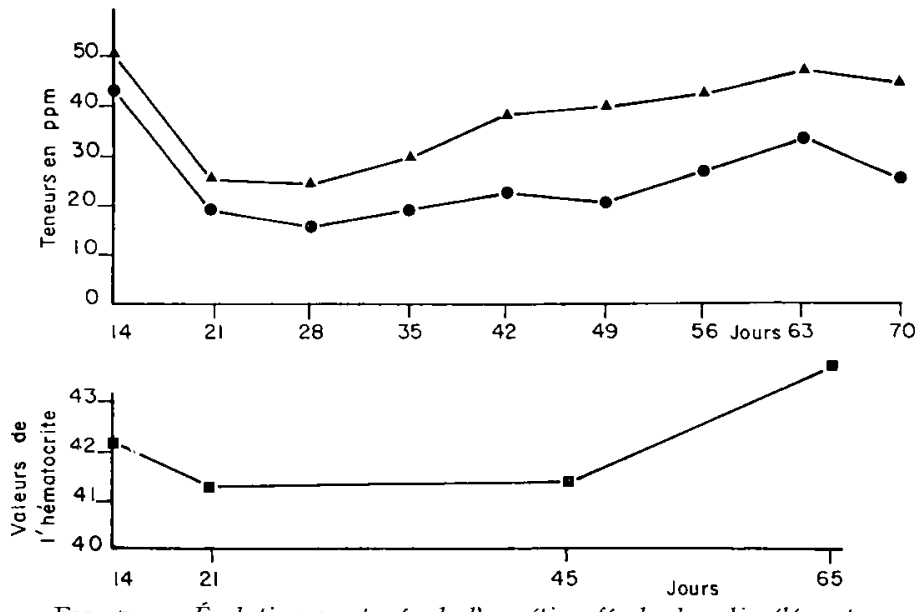

FIG. 7. - Évolution comparée de l'excrétion fécale des oligoéléments et de l'hématocrite pour l'ensemble des régimes selon le temps

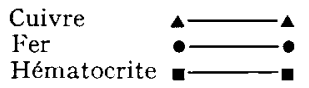

Nous pensons que si les lipides alimentaires ont une influence sur l'excrétion fécale des oligo-éléments c'est parce que les valeurs de 1'hématocrite sont sous la dépendance de ces mêmes lipides. Les lipides qui déterminent un hématocrite élevé, installent de ce fait, une sorte d'état pléthorique en fer, si on se réfère aux relations 
classiquement établies (Widdowson et MaCCANCE, I937; Crosby, I968), on doit avoir dans ce cas une absorption réduite qui s'exprime par une excrétion accrue; c'est ce que nous observons. A l'opposé, les lipides qui induisent de faibles valeurs de l'hématocrite ont tendance à entraînet une meilleure absorption du fer et une diminution de l'excrétion fécale; c'est ce que nous constatons également.

Nous écartons ainsi une hypothèse qui aurait pu être avancée a priori et selon laquelle les lipides potvvaient gêner directement l'absorption intestinale. Il a été avancé que le calcium et le magnésium, complexés à des acides gras saturés sous forme de savons insolubles, avaient une assimilation perturbée ('THIEULIN, I968; TOUl LEC et MathIEU, I969); une telle inhibition de l'absorption pour le fer ne parait pas valable.

Nous devons rechercher ailleurs une explication à la relation très significative, qui existe entre la nature des lipides alimentaires et l'anémie.

\section{3. - Les réserves de fer et de cuivre dans le foie, la rate et les reins}

De l'examen des résultats obtenus, il ressort que les teneurs en fer du foie, de la rate et des reins, évoluent dans le même sens, et ceci en fonction des lipides utilisés dans la ration. Il en est de même pour le cuivre, à l'exception de ce qui concerne la rate.

Il faut cependant ajouter, que de ces trois organes, c'est le foie qui contient le plus de fer et nous limiterons notre discussion à ce qui se passe au niveau hépatique.

Le stockage des oligo-éléments est en relation avec les lipides de l'alimentation. Le saindoux (lot 3), l'huile de colza (lot 4), induisent des réserves hépatiques faibles. Le beurre laitier (lot 5 ) détermine au contraire une mise en réserve hépatique importante. La lécithine du soja (lot I) et l'huile de coprah (lot 2) conduisent à des élats intermédiaires.

Le rapprochement des valeurs de l'hématocrite de la teneur en fer hépatique fait apparaître une liaison très étroite entre ces deux paramètres $(r=+0,8 \mathbf{I} 3)$. Par contre, nous ne trouvons pas de liaison significative entre les valeurs de l'hématocrite et celles du cuivre $(r=+0$, I05). Ceci est à notre avis nullement surprenant si nous considérons, ce qui est ordinairement admis, que le cuivre agit à la façon d'un catalyseur tant dans la mobilisation du fer hépatique que dans la synthèse de l'hémoglobine. Pourvu que les quantités de cuivre soient suffisamment représentées et ne descendent pas en-dessous d'un seuil minimal, sans doute relativement bas, les effets consécutifs à une carence ne se manifestent pas. Nous conclurons en ce qui concerne le cuivre que, même si les lipides interviennent dans l'excrétion fécale et le stockage hépatique, cela ne présente pratiquement pas d'intérêt pour les mécanismes qui nous intéressent dans le cadre de notre expérimentation.

Par contre, la relation entre fer hépatique et hématocrite nous paraît particulièrement intéressante. Elle signifie que la valeur de l'hématocrite est, à son tour, en partie conditionnée par les réserves en fer hépatique. Nous sommes conduits à penser que les lipides interviennent dans le métabolisme du fer au niveau du foie, soit dans l'élaboration des réserves, soit dans la libération du fer stocké. Nous estimerions plutôt que l'intervention des lipides s'exerce sur le processus de stockage ; on ne comprendrait pas en effet, dans le cas de la seconde hypothèse, la liaison étroite existant entre réserves martiales hépatiques et hématocrite. 
Dans les conditions de cette expérience, nous mettons en évidence que les lipides interviennent dans les mécanismes de mise en réserve du fer et du cuivre, essentiellement dans le foie. Il est fort probable que les fluctuations de l'hématocrite, et par conséquent celles de l'excrétion fécale, sont sous la dépendance des variations du stockage hépatique.

\section{4. - Rôle probable des acides gras dans l'utilisation du fer et du cuivre}

La diversité des comportements enregistrés paraît dépendre essentiellement de la composition en acides gras des lipides utilisés. Depuis leur configuration et leur vitesse d'incorporation intestinale, jusqu'à leur remaniement dans le foie, les acides gras peuvent intervenir aux différentes étapes de la dynamique martiale.

Comment peut-on expliquer plus précisément l'incidence des lipides alimentaires sur l'hématopoièse?

Nous avons exclu l'hypothèse d'un ralentissement de la pénétration du fer dans la muqueuse intestinale; il faut donc chercher à d'autres niveaux l'incidence des lipides qui se traduit par une tendance à l'anémie.

Le schéma classique de 1'utilisation du fer fait ressortir deux voies de prise en charge après le passage de la muqueuse intestinale : la voie directe qui, à partir du fer plasmatique en permet l'intégration dans la cellule érythrocytaire, la voie indirecte qui interpose une étape de stockage dans les organes de réserves (CrosBY, I963; NAJEAN, I968); Nous signalerons que la carence en cuivre affecte surtout la voie indirecte (UNDERWOOD, I962). Elle se manifeste au niveau du foie en atténuant 1'intensité de la mobilisation du fer mis en réserve (UNDRITZ, I964), alors qu'elle n'intervient pas dans la captation du fer par les cellules hépatiques (UNDERWOOD, I962).

On ne peut envisager à ce niveau bien précis, une telle carence puisqu'il n'y a pas d'accumulation importante du fer dans le foie ; en effet pour certaines sources lipidiques comme l'huile de colza et le saindoux, les stocks sont faibles et sont associés aux causes de l'anémie (liaison étroite entre l'hématocrite et le fer de réserve dans le cadre de notre expérimentation). Nous réservons donc une action sinon exclusive, tout au moins prépondérante, aux lipides alimentaires sur l'anémie envisagée.

Peut-on déjà observer une action des lipides sur le trajet intracellulaire du fer dans l'enterocyte?

La cellule intestinale est le siège de nombreux mécanismes qui supposent une dépense énergétique, et il est montré que l'absorption du fer se fait de façon active (Underwood, I962; Crosby, I963; Dowle et al., Ig60; Helbock et Saltman, I 967 ). A certains égards on peut envisager une véritable compétition entre les différents mécanismes actifs qui s'exercent dans la cellule. Le passage des acides gras à longue châ̂ne, supérieurs à $\mathrm{C}_{16}$, avec la formation des chylomicrons (FREMONT, I97I ; SAlMON LEGAGNEUR et Friend, I97I ; Vodovar et al., I967), entraîne une dépense énergétique. $\mathrm{Y}$ a-t-il de ce fait, par soustraction d'énergie, un ralentissement de la migration intracellulaire $\mathrm{du}$ fer? Nous pouvons penser à cette hypothèse dans le cas de ces deux graisses riches en acides gras longs, sans toutefois pouvoir apporter d'arguments expérimentaux.

L'étape suivante du métabolisme se situe au niveau du foie. Nous avons écarté précédemment l'hypothèse d'une intervention des acides gras sur la mobilisation du fer stocké. Nous pouvons encore concevoir ici, l'existence d'une compétition dans 
l'utilisation de l'énergie. Dans le foie, la dégradation des acides gras par la voie de la $\beta$ oxydation favorise la production d'ATP par l'intermédiaire du NADH et du $\mathrm{FADH}_{2}$ formés à chaque étape du cycle de dégradation. L'augmentation d'A'TP, ainsi obtenue, entraîne la diminutation d'AMP et favorise indirectement, par la voie des citrates l'activation de l'acetyl CoA carboxylase et la synthèse des acides gras au niveau du foie. Cet effet est d'autant plus marqué que les acides sont longs et saturés. La lipogenèse hépatique serait donc favorisée par les acides palmitique et stéarique, composants majeurs du saindoux. En outre, le remaniement des lipides qui s'opère ainsi, s'accompagne d'une exaltation de la synthèse protidique. En effet les $\alpha$ lipoprotéines qui constituent la majeure partie des lipides pénétrant dans le foie par la voie portale, rejoignent la circulation générale sous forme de $\beta$ lipoprotéines beaucoup plus riches en constituants protéiques. Cette synthèse demande une importante énergie qui est soustraite de celle produite par la $\beta$ oxydation; le bilan global de l'opération (catabolisme lipidique, anabolismes protidique et lipidique) est déficitaire. Ceci peut entrấner une difficulté dans la mise en réserve du fer, processus également consommateur d'énergie (KAUFMANN et al., I964).

On peut ainsi supposer que les formes circulantes du fer sont en plus grandes quantités, et qu'elles entrainent une saturation des agents de transport : les transferrines (NAJEAN, I968). Si on se réfère à KATZ et JANDL, I964, cet état de saturation déterminerait une incorporation plus lente du fer dans les érythrocytes, contrairement aux observations de STANLEy et al., r 969 .

Du travail que nous avons effectué et des hypothèses que nous avons émises, on pourrait résumer la conception que nous avons de l'influence des graisses alimentaires sur l'hématopoièse. Les différents lipides que nous avons utilisés dans nos régimes, n'affectent pas la pénétration du fer dans les enterocytes. Ils interviendraient plutôt sur le trajet intracellulaire qui conduit normalement le métal du pole apical à la membrane basale. Ils seraient aussi concernés dans la mise en réserve au niveau du foie, les effets observés variant selon la nature des graisses. Les stocks martiaux hépatiques sont faibles avec les régimes au saindoux et à l'huile de colza, ils sont élevés avec le beurre laitier. Ainsi l'inhibition de la mise en réserve hépatique provoquerait un ralentissement de la synthèse hémoglobinique.

Il est naturel de penser que la diversité des effets, attribués aux graisses, commencent par l'aptitude à l'absorption de ces dernières. L'exemple du saindoux nous paraît particulièrement intéressant : $d u$ fait de sa structure, sa digestibilité se trouve accrue. L'activité lipasique s'exerçant sur les liaisons esters en position $\alpha$ du glycerol, transforme le triglyceride en monopalmitate (THIEULIN, I968 ; TOULLEC et MATHIEU, I969; SAVARY et al., I957 FLANZY, I97I). Or cette présentation favorise l'absorption des acides gras longs saturés (VoDovar et Flanzy, I966; MATTSON, I964). Il n'est donc pas surprenant que le saindoux manifeste des effets anémiants significatifs d'autant que la formation des chylomicrons, étant le fait des acides gras longs présents dans ce lipide, se trouve exaltée. (Vodovar et FIANZY, I967 ; FreEMAN et GEER, I965). Outre les mécanismes que nous avons développés, nous ne pouvons pas ignorer certains processus particuliers susceptibles d'expliquer l'action déterminante de certains lipides. L'huile de colza pourrait, à notre avis, provoquer l'anémie par le processus suivant : il est établi que le catabolisme des acides gras désaturés aboutit à la formation de quantités élevées de peroxydes dont l'action est connue pour déterminer des perturbations particulièrement agressives dans les réactions oxydatives 
mitochondriales, en cas de déséquilibre avec les antioxydants naturels tels que sélenium et vitamine $\mathrm{E}$. En tout état de cause cette répercussion pourrait porter sur la diminution de la durée de vie des érythrocytes (Aschkenasy, I97I). Cependant 1'huile de colza provoque sans doute des troubles qui affectent un plus grand nombre de mécanismes, ce qui la différencierait d'autres sources lipidiques insaturées en raison de sa forte teneur en acide érucique (RocQuelin et PotTEAU, I968). Cette dernière hypothèse expliquerait le mauvais état général de nos animaux nourris avec ce régime.

Reçu pour publication en juin 1973.

\title{
SUMMARY
}

\author{
INFLUENCES OF LIPID DIETS ON COPPER \\ AND IRON METABOLISMS IN THE LABORATORY RAT
}

Experimental investigations on induced anemia in the rat were carried out on series of I 5 subjects with various lipid diets, in connection with regulation of copper and iron metabolisms. The results show that the dietary lipids according to their nature and fatty acid composition behave differently on the haematopoiesis. More especially, the impact of lipid constituants is located at the level of liver storage of iron and copper upon which depend the variations of haematocrit and those of the fecal excretion of trace element. Thus, dairy butter enhances the increase in the storage of iron in the liver, whereas lard and colza oil induce a drecrease in the hepatic storage. Copra oil and soybean lecithin are intermediate and have no apparent effect on the mechanisms of utilization. Anemic states are only brought about when lard and colza oil are used in the diets.

\section{RÉFÉRENCES BIBLIOGRAPHIQUES}

Aschkenasy A., I97I. Nutrition et hématopoïèse. Monographies des Ann. Nutr. et Alim. C. N. R. S., Paris.

Aschkenasy A., Blapin O., 1959. Sang, 30, p. 574 in Nutrition et hématopoïse. Aschkenasy A. C. N. R. S., I97I, Paris.

BoNv J., Ig66. In vivo interaction of cadmium, copper, zinc and iron in the mouse and the rat. $J$. Nutr., 90, 395-399.

Bozzolo G., 1972. Incidences des constituants lipidiques alimentaires sur les métabolismes martial et cuprique chez le rat et le veau. Thèse de docteur-ingénieur. Univ. P. Sabatier, Toulouse.

Brodan V., Brodanova M., Kuhn E., Kordcac V., Valek J., Ig67. Modification de la résorption du fer dans les voies digestives sous l'influence de l'absorption d'éléments nutritifs. Influence des lipides. Ceskosl Gastroenterol. Vyziva 21, n $6,393-400$.

Charpentier J., I966. Pigmentation du veau de boucherie. Influence d'une supplémentation en fer sur la teneur en fer héminique de quelques muscles. Ann. Zootech., 15, 36I-366.

Charpentier J., ig66. Pigmentation musculaire du veau de boucherie. Amn. Zootech., 15, I8I-I96.

Crosby W. H., I963. The control balance by the intestinal mucosa. Blood. 22, 44I-449.

Crosby W. H., 1968. Intestinal absorption. Section 6 Alimentary Canal, 3, American Physiological Society D. C., Washington.

De Gier J., Van Deenen L. L. M., I96r. Bioch. Biophys, Acta, 49, p. 286. In Nutrition et hématopoïèse. AschKenasy A., C. N. R. S., I97I, p. 25 I.

Dowdle G.B., Schachter D., Schenker H., I960. Active transport of ${ }^{59} \mathrm{Fe}$ by everted segments of rat duodenum. Ann. J. Physiol., 198, 609-6r9.

Eeckhout W., Casteels M., Buysse F., I969a. Influence de la teneur en fer des aliments d'allaitement pour veau à l'engrais sur les éléments figurés du sang, la couleur de la viande et les résultats d'engraissement. Ann. Zootech., 18, 249-26r. 
Eeckhout W., Casteels M., Buysse F., r969 b. L'anémie et la couleur de la viande chez le veau à l'engrais de la race Pie-rouge de la Flandre Orientale. Ann. Zootech., 18, 263-276.

FAust E. S., 1908. Arch. exper. Path. Pharmak., 59, p. I7I. In Nutrition et hématopoièse AschkenAsy A. C. N. R. S., 1971, p. 165.

Flanzy J., 197x. Utilisation digestive des acides gras chez le Porc. Ann. Biol. anim. Bioch. Biophys., 11, p. 3II.

Freeman J. A., Geer J.C., I965. Intestinal fat and iron transport, goblet cell mucus secretions and cellular changes in protein deficiency observed with the electron microscope. Amer. J. of digestive disease, p. 1002-1023.

Freeman L. W., Jonhson V., I940. Amer. J. Physiol., 130, p. 723. In Nutrition et hématopoïèse Aschkenasy A., I971, C. N. R. S., p. 165.

Fremont L., r97i. Le transport des acides gras par la voie lymphatique chez le Porc. Ann. Biol. anim. Bioch. Biophys., 11, 353.

Helbock M., Saltman P., r967. The transport of iron by rat intestine. Bioch. Biophys. Acta., 135, 979-990.

Hirooka M., IKeda H., Rubota H., r968. Effect of dietary fat (soy bean oil) on intestinal absorption in rats. Tokohu. J. Exper. Med. jap., 95, no $3,243^{-252 .}$

KatZ S. H., JANDL J. H., I964. The role of transferrin in the transport of the iron into the developping red cell. In Iron metabolism, an international symposium. Gross Springer Verlag Berlin, I04-II7.

KaufmanN N., KLavins J. V., Kinney Y.D., I964. Depression of liver affinity for iron with iron overload. Proc. Exper. Biol. Med. U.S. A., 120, 81 3-816.

Kayser C., 1963. Physiologie tome III, p. 629. Éd. Med. Flammarion, Paris.

Kent G., Orfei H., Minich J., De la Huerga F. E., i963. Effect of hepatic injuries upon iron storage in the liver. Lab. Invest., 12, I094-I IOI.

Levenson S. M., UPJHON H. I., Schechy T. W., I957. Metabolism, 6, p. 807. In Nutrition et hématopoïèse Aschkenasy A., C. N. R. S., 1971, I63.

Loewy A., 1943. Amer. J. Physiol., 140, p. 230. In Nutrition et hématopoïèse, Aschkenasy A., I97I, I63.

LoISEleUR J., I963. Techniques de laboratoire. Chimie physique, chimie biologique. Masson et Cie, tome I, fasc. 2, Paris.

Longini J., Johnson V., 1943. Amer. J. Physiol., 140, p. 34. In Nutrition et hématopoïèse, Asch Kenasy A., C. N. R. S., I971, I63.

Mac Call M. E., Newman G. E., O'brien J. R. P., Walberg L. S., Witts L. J., I962. Studies on iron metabolism. Brit. J. Nutr., 16, 297-304.

Matrone G., Conley C., Wise G. H., Waugh R. H., I957. A study on iron and copper requirement of dairy calves. Depart. of anim. Nutr. North Carolina. Station Raleight, I437-I447.

Mattson F.H., r964. The digestion and absorption of triglycerides. J. Biol. Chem., 239, 2772-2777.

Meng H. G., Freeman S., I948. J. Lab. Med., 33, I65. In Nutrition et hématopoièse, Aschkenasy A. C. N. R. S., I97I, I65.

Muntwyler E., Hanzel R. F., r933. Action of copper and other elements in iron metabolism. Western Reserve Univ., 13, 845-846.

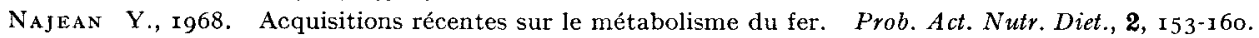

Pinto R., Baldi A., I968. Confronto del conteggio degli eritrociti tra un nuovo diluente fotometrico ed il contaglobuli electronico. Quod. Slavo Diag. 4, 279-28I.

Rocouelin G., Potteau B., 1968. La valeur nutritionnelle et les effets physio-pathologiques de l'huile de colza. Influence de la teneur en acide érucique. Ann. Nutr. Alim., 22, I9I-244.

Rodriguez L., Broglio M., ig68. A simple method for the determination of microhematocrit. Amer. J. Clin. Pathol., 50, 544.

Stanley G., Schad G., Bernier J., Conrad E., ig69. Normal iron absorption in hypertransferrinaemic rats. Brit. J. Haemat., 17, 187 -190.

Salmon Legagneur E., Friend D. W., i971. Digestion des graisses chez la Truie. Ann. Biol. anim. Bioch. Biophys., 11, 312-314.

Savary P., Flanzy J., Desnuelle P., 1957. Emploi de la lipase pancréatique pour l'étude de la structure des corps gras naturels. Bioch. Biophys. Acta. 24, p. 4I4-423.

Thievlin C., 1968. Les divers facteurs influants sur l'utilisation digestive des matières grasses. Ann. Nutr. Alim., 22, 245-258.

Toullec R., MAthieu C. M., I969. Utilisation des matières grasses et de leur principaux acides gras par le veau préruminant à l'engrais. Ann. Biol. anim. Bioch. Biophys., 9, I39-160.

UNDERWOOD E. J., 1962. Trace elements in human and animal nutrition. Acad. Press New York and London.

Undritz E., 1964. Oral treatment of iron deficiency, p. 503-5I7. In Iron metabolism; international symposium Gross Springer Verlag, Berlin.

VAHLQUist B., I946. Formule sanguine chez des nourrissons normaux traités par le fer. Nord Med., 30, Iror. 
Vodovar N., Flanzy J., I966. Mécanisme de la pénétration et voie d'acheminement des graisses dans la cellule absorbante de l'intestin grêle de Porc. Ann. Biol. anim. Bioch. Biophys., 6, 3I5-332.

Vodovar N., Flanzy J., 1967. Voie de pénétration des lipides ingérés dans le stroma des villosités. C. R., Acad. SC. Paris, série D, 264, $850-853$.

Vodovar N., Flanzy J., Françors A. C., I967. Répartition des acides gras ingérés entre vaisseaux sanguins et lactéales centrales dans le stroma des villosités. Ann. Biol. anim. Bioch. Biophys., 7, 423-435.

Watkin D. M., I957. Metabolism, 6, p. 785. In Nutrition et hématopoïèse AschKenasy A., C. N. R. S., I97I, I 65.

Widdowson E. M., MacCance R. A., I937. The absorption and excretion of iron before, during and after a period of very high intake. Bioch. J., 31, 2029. 